\title{
Increased glomerular cell (podocyte) apoptosis in rats with streptozotocin-induced diabetes mellitus: role in the development of diabetic glomerular disease
}

\author{
S. Menini • C. Iacobini • G. Oddi • C. Ricci • \\ P. Simonelli • S. Fallucca • M. Grattarola • F. Pugliese • \\ C. Pesce • G. Pugliese
}

Received: 29 March 2007 / Accepted: 31 July 2007 / Published online: 28 September 2007

(C) Springer-Verlag 2007

\begin{abstract}
Aims/hypothesis Podocyte loss by apoptosis, in addition to favouring progression of established diabetic nephropathy, has been recently indicated as an early phenomenon triggering the initiation of glomerular lesions. This study aimed to assess the rate of glomerular cell death and its relationship with renal functional, structural and molecular changes in rats with experimental diabetes.

Methods Male Sprague-Dawley rats with streptozotocininduced diabetes and coeval non-diabetic control animals were killed at 7 days and at 2, 4 and 6 months for the assessment of apoptosis, renal function, renal structure and the expression of podocyte markers and apoptosis- and cell cycle-related proteins.

Results Glomerular cell apoptosis was significantly increased in diabetic vs non-diabetic rats at 4 months and to an even greater extent at 6 months, with podocytes accounting for $70 \%$ of apoptosing cells. The increase in apoptosis was preceded by increases in proteinuria, albu-
\end{abstract}

Electronic supplementary material The online version of this article (doi:10.1007/s00125-007-0821-y) contains supplementary material, which is available to authorised users.

S. Menini $\cdot$ C. Iacobini $\cdot$ G. Oddi $\cdot$ C. Ricci $\cdot$ P. Simonelli $\cdot$

S. Fallucca $\cdot$ F. Pugliese $\cdot$ G. Pugliese $(\varangle)$

Department of Clinical Sciences, La Sapienza University,

Viale del Policlinico,

155-00161 Rome, Italy

e-mail: giuseppe.pugliese@uniroma1.it

M. Grattarola $\cdot$ C. Pesce

DISTBIMO, University of Genoa Medical School,

Genoa, Italy minuria and mean glomerular and mesangial areas, and by reductions in glomerular cell density and content of synaptopodin and Wilms' tumour protein-1. It coincided with the development of mesangial expansion and glomerular sclerosis, and with the upregulation/activation both of tumour protein $\mathrm{p} 53$, which increased progressively throughout the study, and of p21 (also known as cyclin-dependent kinase inhibitor 1A, CIP1 and WAF1), which peaked at 4 months and decreased thereafter.

Conclusions/interpretation Glomerular cell (podocyte) apoptosis is not an early feature in the course of experimental diabetic glomerulopathy, since it is preceded by glomerular hypertrophy, which may decrease glomerular cell density to the point of inducing compensatory podocyte hypertrophy. This is associated with reduced podocyte protein expression (podocytopathy) and proteinuria, and ultimately results in apoptotic cell loss (podocytopenia), driving progression to mesangial expansion and glomerular sclerosis.

Keywords Apoptosis · Diabetic glomerular disease .

Glomerular hypertrophy · Glomerular sclerosis .

Mesangial expansion $\cdot \mathrm{p} 21 \cdot \mathrm{p} 53 \cdot$ Podocytes .

Proteinuria $\cdot$ Synaptopodin
Abbreviations
DAB 3,3'-diaminobenzidine
fMA fractional mesangial area
GSI glomerular sclerosis index
mGA mean glomerular area
mGV mean glomerular volume
mMA mean mesangial area
PAS periodic acid-Schiff
WT-1 Wilms' tumour protein 1 


\section{Introduction}

Programmed cell death, a normal event in renal tissue homeostasis [1], has been considered a major mechanism for resolution of glomerular hypercellularity in glomerulonephritis $[2,3]$, but also for loss of cellularity and scarring in chronic renal disease [1, 4]. This is suggested by the enhanced apoptosis rate detected in human and experimental renal diseases [5], including diabetic nephropathy [6-8], and by its correlation with loss of renal function and structure [5].

In diabetes, the apoptotic cascade might be specifically triggered by hyperglycaemia and stretching, through pathways involving formation of advanced glycation end products (AGEs) and reactive oxygen species and activation of nuclear factor $\mathrm{kB}$; it is prevented by attenuation of oxidative stress [9-12].

Cell loss by apoptosis is preceded by cell cycle arrest, which may lead to either hypertrophy or death, depending on several factors, including the tumour protein p53/p21 (also known as cyclin-dependent kinase inhibitor 1A, CIP1 and WAF1) pathway [13]. The p53 protein, activated through post-translational modifications [14], induces apoptosis via transcription-dependent and transcriptionindependent mechanisms [15], whereas its downstream target p21 causes growth arrest by inhibiting the activity of the cyclin/cyclin-dependent kinase complexes [16]. Increased glucose- and AGE-induced p53 production and phosphorylation have been reported in mesangial cells [12, 17]. Likewise, p21 upregulation was detected in mesangial cells cultured under high glucose and in glomeruli from diabetic mice [18], whereas diabetic p21-deficient mice exhibited neither glomerular hypertrophy nor proteinuria [19].

Recent evidence has suggested that increased cell death rate in experimental type 1 and 2 diabetes is predominantly accounted for by podocytes, although apoptosis of mesangial and endothelial cells also occurs $[10,11]$. Since podocytes are highly specialised and terminally differentiated epithelial cells, their loss by apoptosis is not compensated for by proliferation and triggers a sequence of events leading to glomerulosclerosis and tubulo-interstitial fibrosis [20, 21], as shown in several forms of focal and segmental glomerulosclerosis [22]. Both cross-sectional [23, 24] and longitudinal $[25,26]$ studies in type 1 and 2 diabetic patients have shown that podocyte depletion is correlated with the degree of albuminuria and disease progression. Recently, reduced podocyte density [27], and also urinary podocyte excretion [28], were found to predict albuminuria more closely than the absolute decrease of podocyte number.

Podocyte loss by apoptosis, in addition to favouring progression of established diabetic nephropathy, has been recently indicated as an early phenomenon playing a central role also in the initiation of glomerular lesions [10].
This study aimed to assess the rate of glomerular cell death in experimental diabetic nephropathy and to identify the cell type(s) involved. In addition, in order to verify the hypothesis that podocytes play a central role in the development of diabetic glomerular disease, we also evaluated the relationship of (podocyte) apoptosis with renal functional and structural changes, as well as the content both of podocyte markers and apoptosis- and cell cycle-related proteins.

\section{Methods}

Experimental design This study was approved by the local ethics committee and carried out in accordance with the Principles of Laboratory Animal Care (NIH Publication no. 85-23, revised 1985) and national laws. Adult (aged 810 weeks) male Sprague-Dawley rats (Harlan Nossan, Milan, Italy) were divided into the following groups: nondiabetic control rats and rats rendered diabetic by streptozotocin $(55 \mathrm{mg} / \mathrm{kg}$ i.p.; Sigma Chemical, St Louis, MO, USA) [29]. Insulin-treated diabetic rats and rats showing spontaneous reversal of diabetes [30] served as control for streptozotocin effect. Rats were studied for 7 days and for 2, 4 and 6 months after initiation of the study ( $n=6$ for both groups at each time point). They were placed in metabolic cages to collect urine; the next day, they were anaesthetised with intramuscular ketamine $(60 \mathrm{mg} / \mathrm{kg}$; Merial Italia, Assago, Milan, Italy) and xylazine $(7.5 \mathrm{mg} / \mathrm{kg}$; Bayer Italia, Milan, Italy), a blood sample was collected and the kidneys were removed. A sagittal slab was cut, fixed in 4\% (vol./vol.) paraformaldehyde and embedded in paraffin. The remaining kidney tissue was used for glomeruli isolation by sequential sieving and total RNA extraction by the guanidine thiocyanatephenol-chloroform method using Trizol reagent (Invitrogen Italia, San Giuliano Milanese, Italy).

Glomerular cell apoptosis Glomerular cell death rate was assessed by immunohistochemistry, as previously reported [11, 31]. Sections were sequentially treated with: (1) Triton $\mathrm{X}-100(0.1 \%$, vol./vol.) for $10 \mathrm{~min}$; (2) hydrogen peroxide in PBS $(0.6 \%$, vol./vol.) for $30 \mathrm{~min}$, to quench endogenous peroxidase activity; (3) serum-free Protein Block (Dako, Glostrup, Denmark) for $10 \mathrm{~min}$, to block non-specific binding; and (4) streptavidin/biotin blocking kit (Vector, Burlingame, CA, USA). Then, sections were incubated overnight at $4{ }^{\circ} \mathrm{C}$ with a rabbit polyclonal antibody against the active form of caspase-3 (Promega, Milan, Italy), followed by a $1 \mathrm{~h}$ incubation with biotinylated goat antirabbit antibody (Dako) and a 30 min incubation with streptavidin-biotinylated horseradish peroxidase complex (StreptABComplex/HRP; Dako) at room temperature. Finally, sections were developed with 3,3'-diaminobenzi- 
dine (DAB, brown colour) in chromogen solution (Liquid $\mathrm{DAB}+$; Dako) for $4 \mathrm{~min}$ and counterstained with haematoxylin. In the negative controls, the primary antibody was omitted. Sections of thymus were used as positive controls. The results were expressed as the percentage of total cells that stained for active caspase-3. The type of glomerular cells undergoing apoptosis was identified both topographically, by counterstaining sections with periodic acid-Schiff (PAS) to mark basement membranes, and by double immunohistochemistry using a goat polyclonal antibody anti-CD31/PECAM-1 (M-20; Santa Cruz Biotechnology, Santa Cruz, CA, USA), a mouse monoclonal antibody against rat Thy-1,1 (Santa Cruz Biotechnology) or a mouse monoclonal antibody anti-synaptopodin (Progen, Wieblingen, Germany) to mark endothelial, mesangial and visceral epithelial cells, respectively. After staining for active caspase-3, cell type-specific antigens were unmasked with Antigen Retrieval Solution, pH 6 (Abcam, Cambridge, UK) or, in the case of CD31/PECAM-1, by enzyme digestion with Antigen Retrieval Kit (Abcam). Then, sections were incubated overnight at $4{ }^{\circ} \mathrm{C}$ with the second primary antibody, followed by a biotinylated anti-mouse IgG (Vector; rat adsorbed) or a biotinylated anti-goat $\operatorname{IgG}$ (Abcam), for $1 \mathrm{~h}$ at room temperature. After a $30 \mathrm{~min}$ incubation with StreptABComplex/HRP, sections were developed for 810 min with Vector SG substrate kit for peroxidase (Vector; blue colour to distinguish from caspase-3 staining), intensified using DAB Enhancing Solution (Vector).

Metabolic parameters Body weights were measured biweekly, whereas blood glucose levels were assessed weekly with an automated colorimetric instrument (Glucocard Memory 2; Menarini Diagnostics, Florence, Italy) from blood obtained by tail venepuncture. Serum AGE levels were assessed by competitive ELISA, using a mouse monoclonal antibody against AGE-modified bovine serum albumin $[11,31]$.

Renal function Serum and urine creatinine levels were measured by the Jaffé method [11, 31]. Total proteinuria was assessed by the Bradford method using the Bradford dye-binding protein assay kit (Pierce Chemical, Rockford, IL, USA). Albuminuria was measured by a kit (Rat Albumin ELISA Quantitation; Bethyl Laboratories, Montgomery, TX, USA). Both values were normalised by the urine creatinine concentration $[11,31]$.

Renal structure Analysis of renal structure was performed by two pathologists blinded to the group assignment. PASstained sections were evaluated for glomerular sclerosis by a standard semiquantitative analysis and expressed as glomerular sclerosis index (GSI), as previously reported $[11,31]$. Morphometrical analysis was performed using a custom-made, C-language macro written with the Optimas 6.5 image analysis system (Optimas, MediaCybernetics, Silver Spring, MD, USA). At least 100 glomerular tuft profiles per sample were evaluated. The number of cells per unit of glomerular tuft area was used as an indirect measure of cell population density [9].Then, the glomerular areas were measured, the harmonic mean of the profile area (mean glomerular area [mGA]) was obtained and the mean glomerular volume $(\mathrm{mGV})$ was estimated from it according to the formula of DeHoff and Rhines [32]. PAS-positive material in each of these glomeruli was quantified and expressed as a percentage of the glomerular tuft area (fractional mesangial area [fMA]). Finally, the mean mesangial (matrix) area (mMA) was calculated by the formula: $(\mathrm{fMA} \times \mathrm{mGA}) / 100[11,31]$.

Podocyte markers The glomerular content of synaptopodin, a component of the foot process cytoskeleton, and of the Wilms' tumour protein-1 (WT-1), a transcription factor participating in podocyte differentiation and maintenance of mature phenotype, was assessed by immunohistochemistry using the anti-synaptopodin antibody indicated above and a rabbit polyclonal anti-WT-1 antibody (Santa Cruz Biotechnology), respectively.

Apoptosis- and cell cycle-related proteins Transcripts of the p53 gene (also known as Tp53) were quantified by competitive RT-PCR [11,31] and results were expressed as the ratio of $p 53$ to $\beta$-actin mRNA levels. The following primers were used: p53: sense 5'-CTGCTTCAGCTA CACCGTGGC-3', antisense 5'-GAGTCAGTGCACATAA CAGAC-3'; and $\beta$-actin: sense 5'-TCT-AGG-CAC-CAAGGT-GTG-3', antisense 5'-TCA-TGA-GGT-AGT-CCGTCA-GG-3'. Protein levels of p21 and activated p53 were assessed by immunohistochemistry using a rabbit polyclonal antibody to p21 (Abcam) and a rabbit polyclonal antibody to phosphorylated (at Ser-15) p53 (Abcam), respectively.

Statistical analysis Values are expressed as means $\pm \mathrm{SD}$; the percentage change vs control was also calculated. Statistical significance was evaluated by Student's $t$ test for comparison between diabetic and non-diabetic rats, and by oneway ANOVA followed by the Student-Newman-Keuls test for comparison of diabetic and non-diabetic rats at different time points.

\section{Results}

Glomerular cell apoptosis Glomerular immunoreactivity for active caspase-3 increased significantly throughout the study in diabetic rats (19.5-fold increment at 6 month vs 


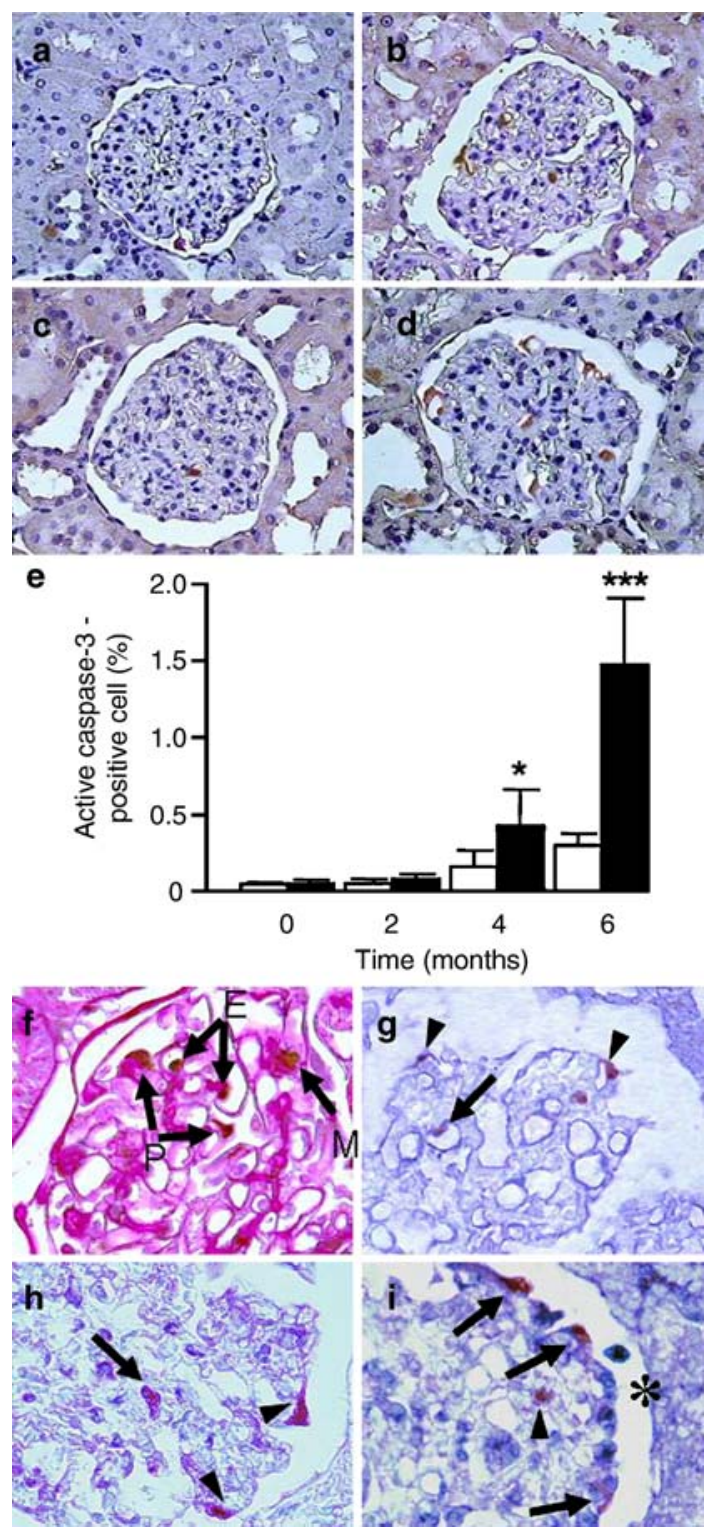

Fig. 1 Immunohistochemistry for active caspase-3 from representative non-diabetic and diabetic rats at 4 months (a, b, respectively) and 6 months (c, d, respectively) of disease duration; $\times 400$ magnification. e Glomerular cell death rate in non-diabetic (open bars) and diabetic (closed bars) rats at times 0 (7 days) and 2, 4 and 6 months of disease duration. ${ }^{*} p<0.05, * * * p<0.001$ vs the corresponding non-diabetic controls. f Topographic identification of glomerular endothelial cells (E), mesangial cells (M) and podocytes (P) by PAS counterstaining; $\mathbf{g}$ double immunohistochemistry for active caspase-3 (brown) plus CD31/PECAM-1 for endothelial cells; $\mathbf{h}$ Thy-1,1 for mesangial cells; and $\mathbf{i}$ synaptopodin for podocytes (blue) (all at $\times 1000$ magnification). The arrows $(\mathbf{f}-\mathbf{i})$ indicate double-positive cells with typical topographical localisation. The arrowheads $(\mathbf{g}-\mathbf{i})$ indicate cells positive for active caspase-3 but not for specific cell markers. The asterisk $\left(^{*}\right)(\mathbf{i})$ marks a podocyte detached from the membrane and lost in the urinary space

2 month time point, $p<0.001)$ and to a much lower extent in non-diabetic animals (5.5-fold increment, $p<0.001$ ). However, the rate of glomerular cell death, which was negligible in both animal groups at 7 days and 2 months, was significantly increased in diabetic vs non-diabetic rats at 4 (2.7-fold) and at 6 months (fivefold) of disease duration (Fig. 1a-e). Topographical identification of apoptosing cell types showed that podocytes accounted for $\sim 70 \%$ of excess cell death in diabetic rats, with the remaining $30 \%$ consisting of mesangial and endothelial cells (Fig. 1f). Double immunohistochemistry provided similar results, although the percentage of apoptosing podocytes was somewhat lower, probably due to the progressive synaptopodin loss (see below) resulting in a certain number of undetermined cells (Fig. 1g-i).

Metabolic parameters Diabetic rats showed impaired body growth, elevated blood glucose levels and progressively increased serum AGE concentrations compared with nondiabetic control animals (Table 1).

Renal function While serum creatinine levels did not differ between the two groups at any time point (Table 1), proteinuria and albuminuria increased progressively with time in non-diabetic $(p<0.01)$ and diabetic $(p<0.001)$ rats; values were significantly higher in diabetic vs non-diabetic animals at all time points (2.0-, 3.1- and 3.6-fold for proteinuria and 1.8-, 2.3- and 2.8-fold for albuminuria, at 2, 4 and 6 months, respectively) (Table 1). No increase in proteinuria and albuminuria vs coeval non-diabetic rats was detected in diabetic rats treated with insulin or in rats showing spontaneous reversal of diabetes (Table 2).

Renal structure Kidney wet weight increased (by 28\%) only in diabetic rats between 4 and 6 months of disease duration (Table 1), although the kidney weight/body weight ratio was higher in diabetic than in non-diabetic animals from 2 months. GSI increased with time in diabetic to a greater degree than in non-diabetic rats (fourfold vs 1.7fold) and was significantly different between the two groups at 4 and 6 months (Fig. 2a). While cell number per glomerulus decreased in diabetic vs non-diabetic rats only at 6 months (not shown), glomerular cell density decreased significantly with time in both groups $(p<0.001)$, with significantly lower levels in diabetic vs non-diabetic rats at all time points $(-19,-29$ and $-32 \%$ at 2,4 and 6 months, respectively) (Fig. 2b). Morphometrical analysis showed a progressive increase of $\mathrm{mGA}, \mathrm{mGV}$ and $\mathrm{mMA}$ in both groups $(p<0.001)$, with significantly higher levels in diabetic vs non-diabetic animals at all time points (23$112 \%$ increase) (Fig. 2c-e). As a consequence, fMA increased with time only in diabetic rats $(p<0.001)$, which exhibited higher values than the non-diabetic controls at 4 (by $15 \%$ ) and 6 (by 67\%) months of disease duration (Fig. 2f). Morphometrical parameters in diabetic rats treated with insulin or showing spontaneous reversal of diabetes were similar to those detected in non-diabetic rats (Table 2). 
Table 1 Values as indicated in non-diabetic and diabetic rats at 7 days (0) and at 2, 4 and 6 months of disease duration

\begin{tabular}{llllllll}
\hline $\begin{array}{l}\text { Rat } \\
\text { group }\end{array}$ & $\begin{array}{l}\text { Final body } \\
\text { weight }(\mathrm{g})\end{array}$ & $\begin{array}{l}\text { Non-fasting } \\
\text { blood glucose } \\
(\mathrm{mmol} / \mathrm{l})\end{array}$ & $\begin{array}{l}\text { Serum AGEs } \\
(\mathrm{U} / \mathrm{ml})\end{array}$ & $\begin{array}{l}\text { Serum creatinine } \\
(\mu \mathrm{mol} / \mathrm{l})\end{array}$ & $\begin{array}{l}\text { Urinary protein/ } \\
\text { creatinine } \\
(\mu \mathrm{g} / \mathrm{mg})\end{array}$ & $\begin{array}{l}\text { Urinary albumin/ } \\
\text { creatinine } \\
(\mu \mathrm{g} / \mathrm{mg})\end{array}$ & $\begin{array}{l}\text { Kidney wet } \\
\text { weight }(\mathrm{g})\end{array}$ \\
\hline ND-0 & $270 \pm 3$ & $4.86 \pm 0.17$ & $0.87 \pm 0.14$ & $29.93 \pm 0.94$ & $2.10 \pm 0.44$ & $1.58 \pm 0.31$ & $1.86 \pm 0.07$ \\
D-0 & $270 \pm 4$ & $23.34 \pm 0.82^{* * *}$ & $0.88 \pm 0.11$ & $29.78 \pm 0.70$ & $2.07 \pm 0.45$ & $1.57 \pm 0.34$ & $1.85 \pm 0.09$ \\
ND-2 & $502 \pm 52$ & $4.75 \pm 0.41$ & $0.99 \pm 0.17$ & $30.08 \pm 0.96$ & $2.42 \pm 0.49$ & $1.76 \pm 0.53$ & $1.89 \pm 0.30$ \\
D-2 & $315 \pm 38^{* * *}$ & $32.15 \pm 1.25^{* * *}$ & $5.93 \pm 0.85^{* * *}$ & $30.23 \pm 1.04$ & $4.89 \pm 0.98 * * *$ & $3.15 \pm 0.61 * * *$ & $1.91 \pm 0.35$ \\
ND-4 & $653 \pm 44$ & $4.88 \pm 0.29$ & $2.19 \pm 0.39$ & $30.15 \pm 1.11$ & $2.94 \pm 0.67$ & $2.03 \pm 0.59$ & $1.75 \pm 0.09$ \\
D-4 & $333 \pm 37 * * *$ & $31.14 \pm 2.30^{* * *}$ & $10.47 \pm 1.24 * * *$ & $30.32 \pm 1.25$ & $8.95 \pm 1.68 * * *$ & $4.73 \pm 1.07 * * *$ & $1.81 \pm 0.37$ \\
ND-6 & $638 \pm 49$ & $5.01 \pm 0.26$ & $2.40 \pm 0.46$ & $30.17 \pm 0.66$ & $3.63 \pm 0.48$ & $2.49 \pm 0.48$ & $1.74 \pm 0.18$ \\
D-6 & $376 \pm 43 * * *$ & $32.40 \pm 1.48 * * *$ & $14.82 \pm 1.10^{* * *} 30.29 \pm 1.92$ & $12.93 \pm 1.11 * * *$ & $6.98 \pm 0.85 * * *$ & $2.32 \pm 0.33 * *$ \\
\hline
\end{tabular}

Values are means $\pm \mathrm{SD} ; n=6$

$N D$ non-diabetic, $D$ diabetic

$* * p<0.01$ and $* * * p<0.001$ for difference between D rats and corresponding ND controls

Podocyte markers Glomerular content of synaptopodin, but not WT-1, decreased significantly with time in non-diabetic rats $(-37 \%, p<0.01)$. Conversely, both proteins decreased in diabetic animals throughout the study $(-56 \%, p<0.001$ and $-29 \%, p<0.05$, respectively), with significantly lower levels vs non-diabetic animals at all time points $(-33$ to $-45 \%$ and -27 to $-40 \%$, respectively) (Figs. 3, 4). No difference in synaptopodin content was observed in rats at 3 to 7 days after streptozotocin injection (Electronic supplementary material [ESM] Fig. 1) or in those in which diabetes was reversed, either spontaneously or due to insulin treatment (not shown), as compared with the corresponding non-diabetic controls.

Apoptosis- and cell cycle-related proteins Glomerular expression of the $p 53$ gene did not change with time in non-diabetic rats, whereas it increased significantly in diabetic animals $(p<0.001)$, with significant differences between the two groups at 4 and 6 months (Fig. 5a), when immunoreactivity for activated p53 was detected only in diabetic glomeruli (ESM Fig. 2). Glomerular levels of p21 protein increased progressively $(p<0.001)$ in non-diabetic and diabetic rats. In the latter group, it peaked at 4 months, when it was 13.6-fold higher than in controls, decreasing thereafter, although at 6 months it was still higher than in non-diabetic animals (+45\%) (Fig. 5b, ESM Fig. 2).

\section{Discussion}

The assessment of glomerular cell death rate in diabetic rats indicated that apoptosis is a central feature of experimental diabetic nephropathy, in keeping with previous reports from

Table 2 Values as indicated in non-diabetic rats, diabetic rats and diabetic rats treated with insulin or showing spontaneous diabetes reversal at 6 months of disease duration

\begin{tabular}{|c|c|c|c|c|c|c|c|c|c|}
\hline $\begin{array}{l}\text { Rat } \\
\text { group }\end{array}$ & $\begin{array}{l}\text { Urinary } \\
\text { protein/ } \\
\text { creatinine } \\
(\mu \mathrm{g} / \mathrm{mg})\end{array}$ & $\begin{array}{l}\text { Urinary } \\
\text { albumin/ } \\
\text { creatinine } \\
(\mu \mathrm{g} / \mathrm{mg})\end{array}$ & $\begin{array}{l}\text { Glomerular } \\
\text { apoptosis } \\
(\%)\end{array}$ & GSI & $\begin{array}{l}\text { Glomerular } \\
\text { cell density } \\
\left(1 / \mathrm{mm}^{2} \times 10^{3}\right)\end{array}$ & $\begin{array}{l}\mathrm{mGA} \\
\left(\mu \mathrm{m}^{2} \times 10^{3}\right)\end{array}$ & $\begin{array}{l}\mathrm{mGV} \\
\left(\mu \mathrm{m}^{3} \times 10^{6}\right)\end{array}$ & $\begin{array}{l}\text { mMA } \\
\left(\mu \mathrm{m}^{2} \times 10^{3}\right)\end{array}$ & $\begin{array}{l}\mathrm{fMA} \\
(\%)\end{array}$ \\
\hline ND & $3.63 \pm 0.48$ & $2.49 \pm 0.48$ & $0.29 \pm 0.08$ & $0.43 \pm 0.08$ & $6.05 \pm 0.51$ & $13.4 \pm 0.7$ & $1.17 \pm 0.09$ & $2.46 \pm 0.12$ & $18.43 \pm 1.35$ \\
\hline $\mathrm{D}$ & $12.93 \pm 1.11^{* * *}$ & $6.98 \pm 0.85^{* * *}$ & $1.46 \pm 0.44 * * *$ & $1.19 \pm 0.16^{* * *}$ & $4.12 \pm 0.45^{* * *}$ & $17.0 \pm 0.8 * * *$ & $1.67 \pm 0.12 * * *$ & $5.23 \pm 0.62 * * *$ & $30.73 \pm 3.12 * * *$ \\
\hline DI & $4.03 \pm 0.79$ & $2.88 \pm 0.47$ & $0.34 \pm 0.18$ & $0.46 \pm 0.08$ & $5.78 \pm 0.14$ & $14.1 \pm 0.4$ & $1.25 \pm 0.06$ & $2.69 \pm 0.24$ & $19.18 \pm 2.29$ \\
\hline DR & $3.96 \pm 1.02$ & $2.79 \pm 0.66$ & $0.31 \pm 0.09$ & $0.42 \pm 0.11$ & $5.70 \pm 0.24$ & $13.9 \pm 0.3$ & $1.23 \pm 0.04$ & $2.43 \pm 0.13$ & $17.53 \pm 1.25$ \\
\hline
\end{tabular}

Values are means $\pm \mathrm{SD} ; n=6$

$N D$ non-diabetic rats, $D$ diabetic rats, $D I$ diabetic rats treated with insulin, $D R$ rats showing spontaneous diabetes reversal

$* * * p<0.001$ vs the corresponding ND rats 
Fig. 2 GSI (a), glomerular cell density (b), mGA (c), mGV (d), mMA (e) and fMA (f) in nondiabetic (open bars) and diabetic (closed bars) rats at times 0 (7 days) and 2, 4 and 6 months of disease duration. ${ }^{*} p<0.05$, $* * p<0.01, * * * p<0.001$ vs the corresponding non-diabetic control rats

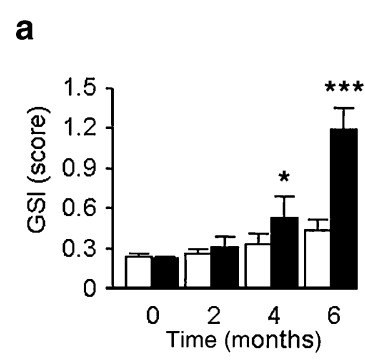

c

e

b

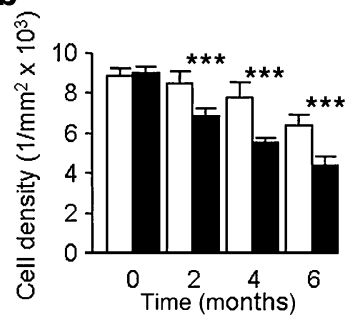

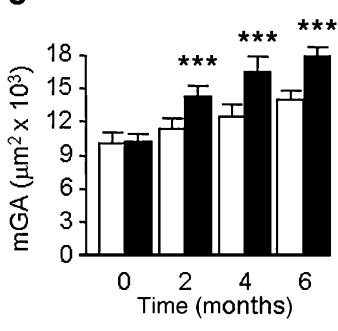

d

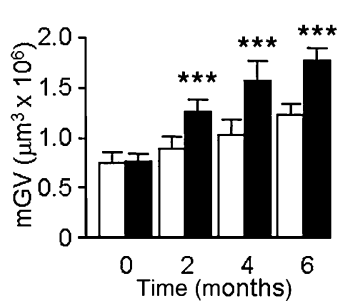

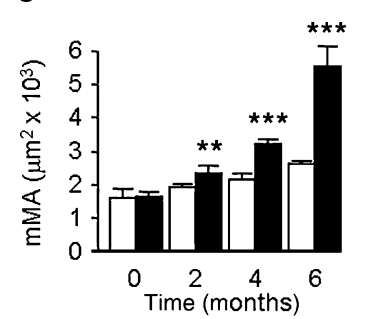

f

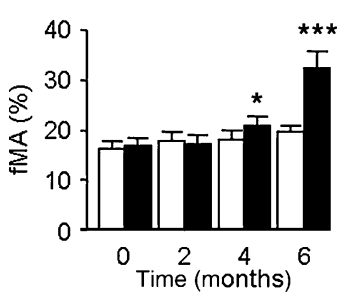

our group $[7,11]$ and other investigators $[8,10]$. This is at variance with glomerular cell proliferation, which was not significantly affected in this experimental animal model of diabetes [7, 30]. Topographical localisation and double immunohistochemistry also indicated that podocytes are the predominant apoptosing cells under these conditions, although endothelial and mesangial cells are also lost. This confirms and extends previous observations [10], which identified the cell type(s) undergoing apoptosis only topographically, and is also consistent with the finding that significant podocyte loss occurs in diabetic patients in parallel with progression of renal disease [23-26], although a relevant role for mesangial cell apoptosis cannot be ruled out.

Our main finding is the demonstration that podocyte loss by apoptosis (podocytopenia) is not an early phenomenon, since it becomes detectable at 4 months and is particularly pronounced only at 6 months of disease duration, being preceded by reduction of podocyte proteins suggesting podocyte injury (podocytopathy). This is in contrast with the recent study from Susztak et al. [11], who found a sharp increase in glomerular cell death rate by the TUNEL reaction in 4 week Akita and 8 week $d b / d b$ mice, whereas it is in keeping with a previous report [8] on the $d b / d b$ mouse model, which detected significant apoptosis, as assessed by caspase-3 cleavage and TUNEL reaction, in 16 week, but not 8 week animals. Thus, although the differences in the experimental models used may be important, we believe that the discrepancy between our results and others [11] may be explained, at least partly, by the higher specificity for apoptosis of caspase-3 cleavage/activation, as compared with nuclear fragmentation by the TUNEL method, which also detects necrotic cells. In addition, Susztak et al. [11] confirmed the early onset of podocyte apoptosis by showing a significant reduction of podocyte number, as assessed by synaptopodin and WT-1 staining. However, this

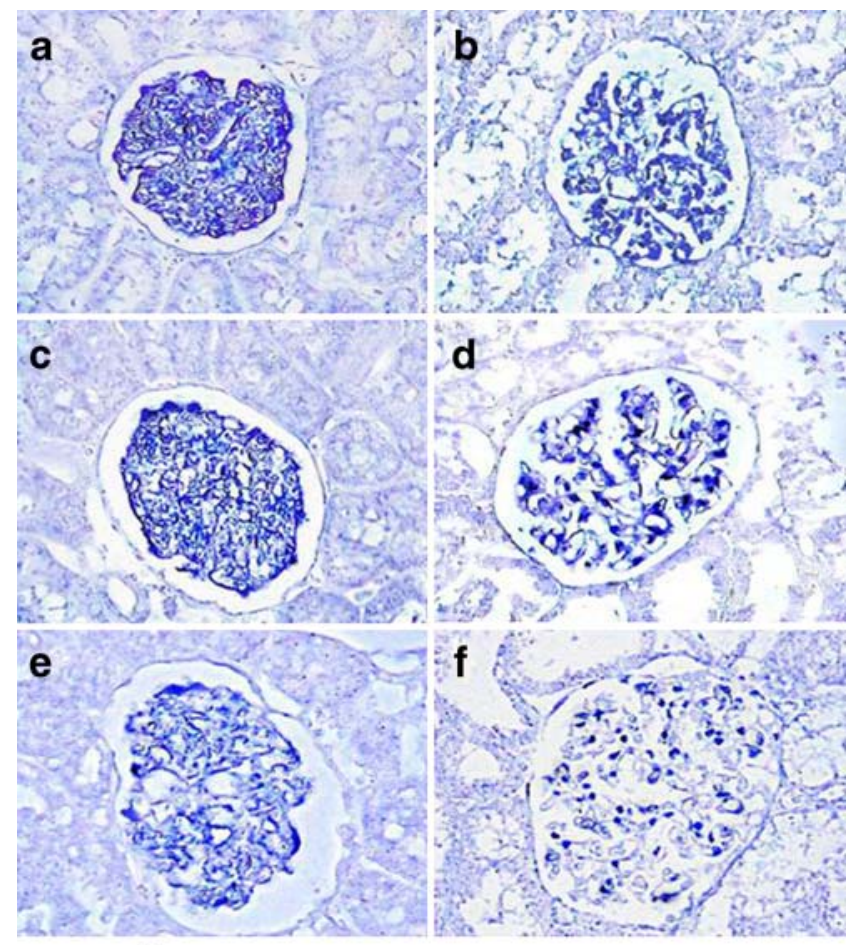

g

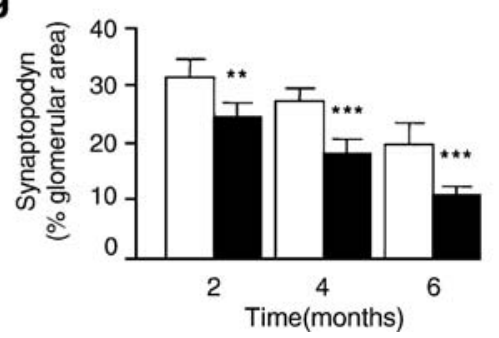

Fig. 3 Immunohistochemistry for synaptopodin from representative non-diabetic (a) and diabetic (b) rats at 2, 4 (c, d, respectively) and 6 months (e, f, respectively) of disease duration. Vector SG (blue) was used as peroxidase substrate, $\times 400$ magnification. $g$ Quantification of glomerular synaptopodin expression in non-diabetic (open bars) and diabetic (closed bars) rats at 2, 4 and 6 months of disease duration. ${ }^{* *} p<0.01, * * * p<0.001$ vs corresponding non-diabetic controls 


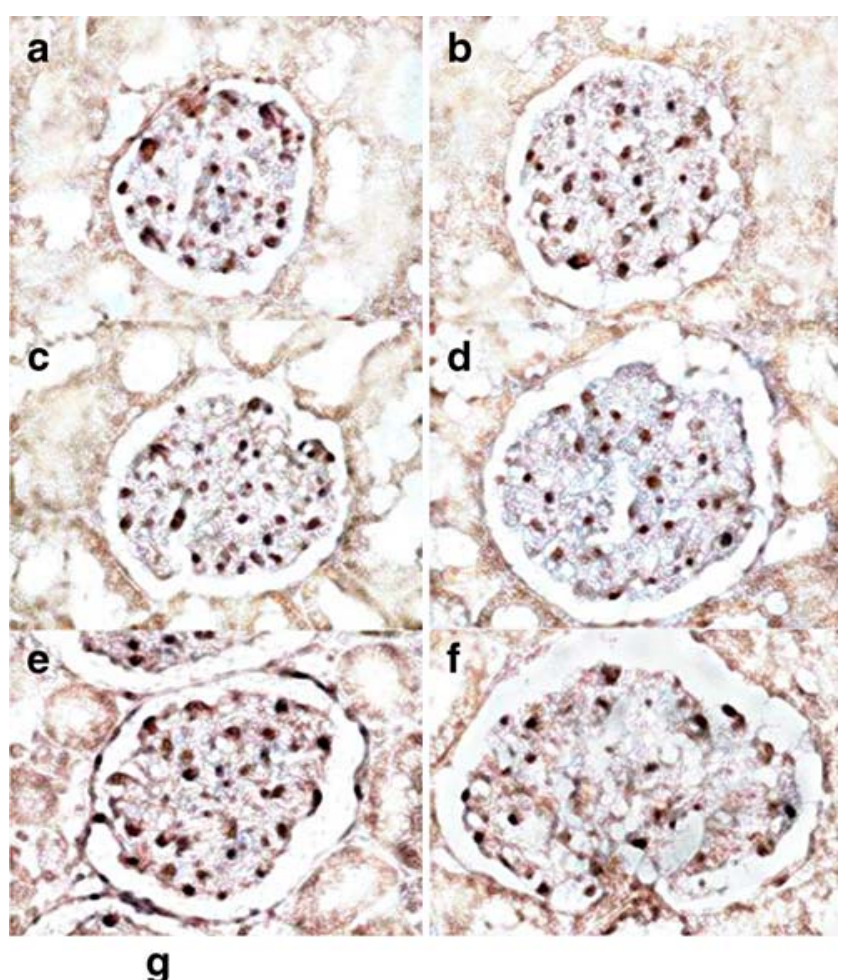

g

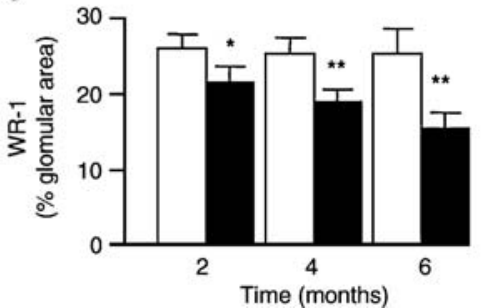

Fig. 4 Immunohistochemistry for WT-1 from representative nondiabetic (a) and diabetic (b) rats at 2, 4 (c, d, respectively) and 6 months (e, f, respectively) of disease duration. DAB (brown) was used as peroxidase substrate, $\times 400$ magnification. $g$ Quantification of glomerular WT-1 expression in non-diabetic (open bars) and diabetic (closed bars) rats at 2, 4 and 6 months of disease duration. ${ }^{*} p<0.05$, $* * p<0.01$ vs the corresponding non-diabetic controls

method cannot be considered to be a surrogate marker of podocyte loss (podocytopenia), but rather an index of podocyte injury (podocytopathy), as shown also by our data.

The concurrent assessment of changes in renal function and structure allowed us to compare the time course of apoptotic phenomena with that of events that mark the development and progression of renal disease in experimental diabetes.

Proteinuria, albuminuria, increased glomerular and mesangial areas, and reduced glomerular cell density were observed in diabetic rats from 2 months of disease duration, much earlier than the time when significant apoptosis was detected, which coincided with the initial increase in GSI and fMA. Thus, the reduced cell density was relative to the increase of filtration surface area. Taken together, these findings indicate that glomerular hypertrophy is the initial event in the pathogenetic sequence leading to the development of diabetic glomerulopathy, although podocytes participate in this cascade by undergoing sequential alterations as a consequence of glomerular enlargement. By increasing the filtration surface area, glomerular hypertrophy produces a similar phenotype to that seen with podocyte depletion, as reported in the aging rat [33]. To occupy this increased area, podocytes, which are not capable of proliferating, undergo a compensatory hypertrophy, which, according to the degree of glomerular enlargement and increase of filtration surface, progresses toward podocyte loss and glomerulosclerosis through the stages of 'nonstressed', 'adaptive' and 'decompensated' cell hypertrophy. This transition to podocytes loss is accompanied by progressive reduction of expression of nephrin and other podocyte proteins, as well as enlargement and effacement/ fusion of foot processes and proteinuria. In our study, in fact, altered podocyte machinery, as indicated by the reduced synaptopodin and WT-1 content, paralleled (and probably contributed to) proteinuria and preceded podocyte loss, which occurred later in the course of the disease and to a much lower extent. In this scenario, the finding that the development of the characteristic structural features of diabetic glomerulopathy, such as glomerular sclerosis and mesangial expansion, was coincident with the occurrence of significant podocyte apoptosis suggests that podocyte loss following compensatory hypertrophy plays a central role in

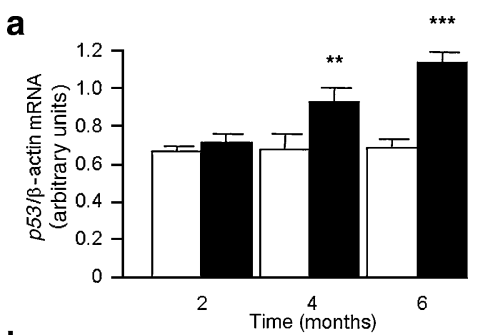

b

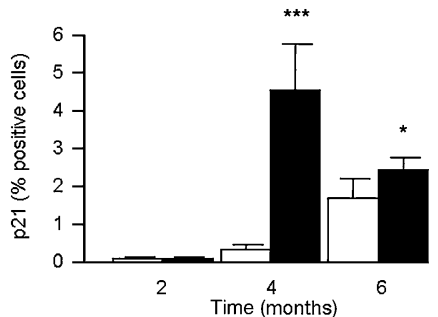

Fig. 5 a Kidney cortex expression of p53 mRNA in non-diabetic (white bars) and diabetic (black bars) rats at 2, 4 and 6 months of disease duration, normalised to $\beta$-actin (by dividing the ratio of $p 53$ $\mathrm{cDNA} /$ mutant [created by introducing a deletion into the original PCR product] by the ratio of $\beta$-actin cDNA/mutant according to the optical densities determined by scanning densitometry). ${ }^{* *} p<0.01$ and $* * * p<0.001$ for difference between diabetic rats and corresponding non-diabetic controls. b Glomerular p21 content in non-diabetic (open bars) and diabetic (closed bars) rats at 2, 4 and 6 months of disease duration. ${ }^{*} p<0.05, * * * p<0.001$ vs the corresponding non-diabetic controls 
the progression from initial glomerular enlargement to the more advanced stages of the disease.

This interpretation, which is consistent with the observation that decreased podocyte density was a better predictor of albuminuria than reduced podocyte number in type 2 diabetic patients [27], is further supported by the observation that the same sequence of events also occurred in the non-diabetic rats with increasing age, though to a much lesser extent, as initially reported [33]. In fact, both the glomerulus and the mesangium became progressively more enlarged in parallel with the increase of proteinuria and reduction of glomerular cell density and synaptopodin expression. Likewise, the increment in glomerular cell apoptosis was coincident with the increase in GSI and mesangial expansion. The unchanged cell death rate and synaptopodin expression at the time of diabetes induction and in rats that were treated with streptozotocin, but subsequently experienced a reversal of diabetes, either spontaneously or due to insulin administration, ruled out the possibility that protein and podocyte loss were caused by streptozotocin.

In diabetic animals, while p21 reached the maximal level at 4 months and decreased thereafter, $p 53$ mRNA and Ser15 -phosphorylated p53 protein continued to rise during the 6 month period. These different profiles could be explained by the fact that, although $\mathrm{p} 21$ is a downstream target of $\mathrm{p} 53$, it is also transcriptionally activated by $\mathrm{p} 53$-independent mechanisms, involving other transcription factors and tumour suppressors that are activated or upregulated in diabetic nephropathy and participate in the pathogenesis of glomerular hypertrophy [34, 35]. These alternative pathways of p21 activation could prevail over the p53dependent mechanism at 4 months, when p53 is only modestly upregulated. The trend of p53 and p 21 upregulation is consistent with the finding that cell loss through apoptosis was preceded by glomerular enlargement, mainly due to TGF- $\beta$ - and p21-dependent cell hypertrophy [36], although other factors might also be involved, particularly in the early stages when p21 is not yet upregulated. Subsequently, the increased p53/p21 ratio and the activation of p53 through phosphorylation at Ser-15 observed at 6 months, together with changes involving the pattern of mitogen-activated protein kinase activation and the extent of reactive oxygen species production [37, 38], could have driven the apoptotic phenomena. This view is in keeping with our previous report that ablation of $\mathrm{p} 66^{\text {Shc }}$, which is stabilised by p53 [39], prevented diabetic glomerulopathy in parallel with attenuation of diabetes-induced oxidative stress and glomerular cell apoptosis [11].

In conclusion, this study showed that podocyte apoptosis is not an early feature in the course of experimental diabetic glomerulopathy, since it is preceded by glomerular hypertrophy, which may decrease glomerular cell density to the point of inducing sequential hypertrophic changes of podocytes associated with proteinuria and reduced podocyte proteins (podocytopathy). This process ultimately results in podocyte loss by apoptosis (podocytopenia), which drives progression from initial glomerular enlargement to mesangial expansion and glomerular sclerosis.

Acknowledgements This work was supported by grants from the Ministry of University and Scientific and Technological Research of Italy ( $40 \%$ and $60 \%$ ), the Ministry of Health of Italy and the Diabetes, Endocrinology and Metabolism Foundation, Rome, Italy.

Duality of interest The authors declare that there is no duality of interest associated with this manuscript.

\section{References}

1. Savill J (1999) Regulation of glomerular cell number by apoptosis. Kidney Int 56:1216-1222

2. Baker AJ, Mooney A, Hughes J, Lombardi D, Johnson RJ, Savill J (1994) Mesangial cell apoptosis: the major mechanism for resolution of glomerular hypercellularity in experimental mesangial proliferative nephritis. J Clin Invest 94:2105-2116

3. Shimizu A, Kitamura H, Masuda Y, Ishizaki M, Sugisaki Y, Yamanaka N (1995) Apoptosis in the repair process of experimental proliferative glomerulonephritis. Kidney Int 47: $114-121$

4. Ortiz A, Lorz C, Justo P, Catalán MP, Egido J (2001) Contribution of apoptotic cell death to renal injury. J Cell Mol Med 5:18-32

5. Sugiyama H, Kashihara N, Makino H, Yamasaki Y, Ota A (1996) Apoptosis in glomerular sclerosis. Kidney Int 49:103-111

6. Zhang W, Khanna P, Chan LL, Campbell G, Ansari NH (1997) Diabetes-induced apoptosis in rat kidney. Biochem Mol Med 61:58-62

7. Pesce C, Menini S, Pricci F et al (2002) Glomerular cell replication and cell loss through apoptosis in experimental diabetes mellitus. Nephron 90:484-488

8. Mishra R, Emancipator SN, Kern T, Simonson MS (2005) High glucose evokes an intrinsic proapoptotic pathway in mesangial cells. Kidney Int 57:82-93

9. Odetti P, Pesce C, Traverso N et al (2003) Comparative trial of $\mathrm{N}$-acetyl-cysteine, taurine, and oxerutin on skin and kidney damage in long-term experimental diabetes. Diabetes 52:499-505

10. Susztak K, Raff AC, Schiffer M, Bottinger EP (2006) Glucoseinduced reactive oxygen species cause apoptosis of podocytes and Wpodocyte depletion at the onset of diabetic nephropathy. Diabetes 55:225-233

11. Menini S, Amadio L, Oddi G et al (2006) Deletion of $p 66^{\text {She }}$ longevity gene protects against experimental diabetic glomerulopathy by preventing diabetes-induced oxidative stress. Diabetes 55:1642-1650

12. Kang BPS, Frencher S, Reddy V, Kessler A, Malhotra A, Meggs LG (2003) High glucose promotes mesangial cell apoptosis by oxidant-dependent mechanism. Am J Physiol 284:F455-F466

13. Allen DA, Yaqoob MM, Harwood SM (2005) Mechanisms of high glucose-induced apoptosis and its relationship to diabetic complications. J Nutr Biochem 16:705-713

14. Appella E, Anderson CW (2001) Post-translational modifications and activation of $\mathrm{p} 53$ by genotoxic stresses. Eur J Biochem 268:2764-2772

15. Fridman JS, Lowe SW (2003) Control of apoptosis by p53. Oncogene 22:9030-9040 
16. Ekholm SV, Reed SI (2000) Regulation of G(1) cyclin-dependent kinases in the mammalian cell cycle. Curr Opin Cell Biol 12:676-684

17. Yamagishi S, Inagaki $Y$, Okamoto $T$ et al (2002) Advanced glycation end product-induced apoptosis and overexpression of vascular endothelial growth factor and monocyte chemoattractant protein-1 in human-cultured mesangial cells. J Biol Chem 277:20309-20315

18. Kuan CJ, al-Douahji M, Shankland SJ (1998) The cyclin kinase inhibitor p21WAF1, CIP1 is increased in experimental diabetic nephropathy: potential role in glomerular hypertrophy. J Am Soc Nephrol 9:986-993

19. Al-Douahji M, Brugarolas J, Brown PA, Stehman-Breen CO, Alpers CE, Shankland SJ (1999) The cyclin kinase inhibitor $\mathrm{p} 21^{\mathrm{WAF} 1 / \mathrm{CIP} 1}$ is required for glomerular hypertrophy in experimental diabetic nephropathy. Kidney Int 56:1691-1699

20. Kriz W, LeHir M (2005) Pathways to nephron loss starting from glomerular diseases-insights from animal models. Kidney Int 67:404-419

21. Kriz W, Hartmann I, Hosser H et al (2001) Tracer studies in the rat demonstrate misdirected filtration and peritubular filtrate spreading in nephrons with segmental glomerulosclerosis. J Am Soc Nephrol 12:496-506

22. Kriz W, Hosser H, Hahnel B, Gretz N, Provoost AP (1998) From segmental glomerulosclerosis to total nephron degeneration and interstitial fibrosis: a histopathological study in rat models and human glomerulopathies. Nephrol Dial Transplant 13:2781-2798

23. Steffes MW, Schmidt D, McCrery R, Basgen JM; International Diabetic Nephropathy Study Group (2001) Glomerular cell number in normal subjects and in type 1 diabetic patients. Kidney Int 59:2104-2113

24. Pagtalunan ME, Miller PL, Jumping-Eagle S et al (1997) Podocyte loss and progressive glomerular injury in type II diabetes. J Clin Invest 99:342-348

25. White KE, Bilous RW, Marshall SM et al (2002) Podocyte number in normotensive type 1 diabetic patients with albuminuria. Diabetes 51:3083-3089

26. Meyer TW, Bennett PH, Nelson RG (1999) Podocyte number predicts long-term urinary albumin excretion in Pima Indians with type II diabetes and microalbuminuria. Diabetologia 42:1341-1344

27. Dalla Vestra M, Masiero A, Roiter AM, Saller A, Crepaldi G, Fioretto P (2003) Is podocyte injury relevant in diabetic nephropathy? Studies in patients with type 2 diabetes. Diabetes 52:1031-1035

28. Nakamura T, Ushiyama C, Suzuki S et al (2000) Urinary excretion of podocytes in patients with diabetic nephropathy. Nephrol Dial Transplant 15:1379-1383

29. Pugliese G, Pricci F, Leto G et al (2000) The diabetic milieu modulates the advanced glycation end product-receptor complex in the mesangium by inducing or upregulating galectin-3 expression. Diabetes 49:1249-1257

30. Pugliese G, Pricci F, Pesce C et al (1997) Early, but not advanced, glomerulopathy is reversed by pancreatic islet transplants in experimental diabetic rats: correlation with glomerular extracellular matrix mRNA levels. Diabetes 46:1198-1206

31. Iacobini C, Menini S, Oddi G et al (2004) Galectin-3/AGEreceptor 3 knockout mice show accelerated AGE-induced glomerular injury. Evidence for a protective role of galectin-3 as an AGE-receptor. FASEB J 18:1773-1775

32. DeHoff RT, Rhines FN (1968) Quantitative microscopy. McGrawHill, New York, pp 33-130

33. Wiggins JE, Goyal M, Sanden SK et al (2005) Podocyte hypertrophy, "adaptation," and "decompensation" associated with glomerular enlargement and glomerulosclerosis in the aging rat: prevention by calorie restriction. J Am Soc Nephrol 16:29532966

34. Ruan XZ, Varghese Z, Powis SH, Moorhead JF (2005) Nuclear receptors and their coregulators in kidney. Kidney Int 68:2444-2461

35. Flyvbjerg A (2000) Putative pathophysiological role of growth factors and cytokines in experimental diabetic kidney disease. Diabetologia 43:1205-1223

36. Wolf G (2000) Cell cycle regulation in diabetic nephropathy. Kidney Int 77:S59-S66 (Suppl)

37. Brown L, Benchimol S (2006) The involvement of MAPK signaling pathways in determining the cellular response to p53 activation: cell cycle arrest or apoptosis. J Biol Chem 281:3832-3840

38. Boonstra J, Post JA (2004) Molecular events associated with reactive oxygen species and cell cycle progression in mammalian cells. Gene 337:1-13

39. Trinei M, Giorgio M, Cicalese A et al (2002) A p53-p66 ${ }^{\text {She }}$ signalling pathway controls intracellular redox status, levels of oxidation-damaged DNA and oxidative stress-induced apoptosis. Oncogene 21:3872-3878 